\title{
Foxd1 is an upstream regulator of the renin-angiotensin system during metanephric kidney development
}

\author{
Renfang Song ${ }^{1}$, Maria Luisa S. Sequeira Lopez ${ }^{2}$ and Ihor V. Yosypiv ${ }^{1}$
}

BACKGROUND: We tested the hypothesis that Foxd1, a transcription factor essential for normal kidney development, is an upstream regulator of the renin-angiotensin system (RAS) during ureteric bud (UB)-branching morphogenesis.

METHODS: UB branching, RAS gene, and protein expression were studied in embryonic mouse kidneys. RAS mRNA expression was studied in mesenchymal MK4 cells.

RESULTS: The number of UB tips was reduced in Foxd1 ${ }^{-1-}$ compared with that in Foxd $1^{+/+}$metanephroi on embryonic day E12.5 (14 \pm 2.1 vs. $28 \pm 1.3, P<0.05$ ). Quantitative real-time reverse-transcription polymerase chain reaction (qRT-PCR) demonstrated that renin, angiotensin I-converting enzyme (ACE), and angiotensin (Ang) II receptor type 1 (AT $\left.{ }_{1} R\right)$ mRNA levels were decreased in Foxd $1^{-1-}$ compared with those in Foxd ${ }^{+/+}$E14.5 metanephroi. Western blot analysis and immunohistochemistry showed decreased expression of AGT and renin proteins in Foxd $1^{-1-}$ metanephroi compared with that in Foxd1 $1^{+/+}$metanephroi. Foxd1 overexpression in mesenchymal MK4 cells in vitro increased renin, $A G T, A C E$, and $A T_{1} R$ mRNA levels. Exogenous Ang II stimulated UB branching equally in whole intact E12.5 Foxd1 ${ }^{-/-}$and Foxd1 ${ }^{+/+}$ metanephroi grown ex vivo $(+364 \pm 21 \%$ vs. $+336 \pm 18 \%$, $P=0.42)$.

CONCLUSION: We conclude that Foxd1 is an upstream positive regulator of RAS during early metanephric development and propose that the cross-talk between Foxd1 and RAS is essential in UB-branching morphogenesis.

$\mathbf{T}$ he metanephric kidney is formed via reciprocal interactions of transcription and growth factors expressed in the metanephric mesenchyme, stroma, and ureteric bud (UB) (1). The stroma is a complex mixture of cell types that do not differentiate into nephrons or UBs. The stroma gives rise to the interstitium in the mature kidney, which contains fibroblasts, lipid-laden interstitial cells, lymphocyte-like cells, pericytes, and extracellular matrix $(2,3)$. Recent studies demonstrated that renal stroma is an essential regulator of both UB-branching morphogenesis and nephrogenesis, and provided important insights into the mechanisms by which the stroma regulates metanephric organogenesis. With respect to UB and UB-derived renal collecting system development, inactivation of a number of genes expressed distinctly in the stroma in mice is associated with reduced UB branching or medullary hypodysplasia (Table 1).

The critical role of the renin-angiotensin system (RAS) in UB morphogenesis and renal collecting system development is evident from (i) reduced UB branching observed in angiotensinogen $(A G T)^{-1-}$ and angiotensin (Ang) II $A T_{2}$ receptor $\left(A T_{2} R\right)^{-1-}$ mice or during pharmacologic inhibition of Ang II receptor type $1\left(\mathrm{AT}_{1} \mathrm{R}\right)$ in embryonic metanephroi grown ex vivo, and (ii) occurrance of medullary hypoplasia in mice and renal tubular dysgenesis in humans with mutations in $A G T$, renin, angiotensin-converting enzyme (ACE), or $A T_{1} R$ genes (Table 1) (4). Using immunohistochemical analysis, we previously demonstrated that $\mathrm{AGT}$ and $\mathrm{AT}_{1} \mathrm{R}$ proteins are expressed in both the UB and the surrounding stroma as early as embryonic (E) day E12 in mice (5). Recent studies in renin knock-in reporter mice have demonstrated that juxtaglomerular renin-producing cells originate from the metanephric mesenchyme/stroma on E11-E12, at a time when UB branching is just beginning (6). This raises the intriguing possibility that Ang II can be generated locally in the stroma and then acts in a paracrine manner on the adjacent $\mathrm{AT}_{1} \mathrm{R}$-expressing UBs to induce branching.

In this work, we tested the hypothesis that Foxd1, a forkhead box transcription factor expressed in the stroma, which is essential for normal kidney development $(7,8)$, is an upstream regulator of RAS during UB-branching morphogenesis. We demonstrate that reduced UB branching observed in Foxd1 $1^{-1-}$ metanephroi is accompanied by decreased expression of $\mathrm{AGT}$, renin, $\mathrm{ACE}$, and $\mathrm{AT}_{1} \mathrm{R}$ mRNA as well as AGT and renin protein levels. Conversely, Foxd1 overexpression in mesenchymal cells increases AGT, renin, ACE, and $\mathrm{AT}_{1} \mathrm{R}$ mRNA levels. We propose that the cross-talk between RAS and Foxd1 has an important role in UB-branching morphogenesis.

\section{METHODS}

\section{Metanephric Organ Culture}

Foxd1-mutant mice (7) were acquired from Jackson laboratories, Bar Harbor, ME. Hoxb $7 \mathrm{GFP}+1-$ transgenic mice, which express green fluorescent protein (GFP) exclusively in the UB, were a kind gift

\footnotetext{
${ }^{1}$ Division of Pediatric Nephrology, Department of Pediatrics, Hypertension and Renal Center of Excellence, Tulane University Health Sciences Center, New Orleans, Louisiana ${ }^{2}$ Department of Pediatrics, University of Virginia School of Medicine, Charlottesville, Virginia. Correspondence: Ihor V. Yosypiv (iiosipi@tulane.edu)

Received 24 February 2017; accepted 21 June 2017; advance online publication 2 August 2017. doi:10.1038/pr.2017.157
} 
Table 1. Effect of stromal factors on UB-branching morphogenesis.

\begin{tabular}{|c|c|c|c|}
\hline Gene & Renal phenotype & UB phenotype & References \\
\hline Foxd1 $1^{-/-}$ & Hypoplasia & Reduced branching & (6) \\
\hline$F G F 7^{-1-}$ & Hypoplasia & Reduced branching/size & (17) \\
\hline$R A R \beta 2^{-/-} / R A R a^{-/-}$ & Hypoplasia & Reduced branching & (28) \\
\hline Pod1 ${ }^{-1-}$ & Hypoplasia, dysplasia & Decreased branching, aberrant patterning & (29) \\
\hline$p 57^{\text {Kip2-1- }}$ & Medullary, dysplasia & & (19) \\
\hline$A G T^{\prime-}$ & $\begin{array}{l}\text { Thin medulla, atrophic papilla, hydronephrosis, decreased } \\
\text { UB branching }\end{array}$ & & $(14,32)$ \\
\hline$A T_{1} R^{-/-}$ & Atrophic papilla, hydronephrosis, decreased ureteral peristalsis & & $(33,34)$ \\
\hline
\end{tabular}

UB, ureteric bud.

from F. Costantini (Columbia University, New York, NY). The day when the vaginal plug was observed was considered to be E0.5. Embryonic metanephroi were dissected from double-transgenic Foxd1-mutant;Hoxb $7^{\mathrm{GFP}+}$ mice on E11.5 or E12.5 and grown on polycarbonate transwell filters (Corning Costar, Corning, NY, USA, $0.5 \mu \mathrm{m}$ ) inserted into six-well plates containing Dulbecco's modified essential medium/F12 medium (Gibco BRL, Grand Island, NY, USA) and $0.5 \%$ fetal bovine serum (FBS) or medium, $0.5 \%$ FBS, and Ang II $\left(10^{-5} \mathrm{M}\right)$ for $24 \mathrm{~h}$ at $37^{\circ} \mathrm{C}$ and $5 \% \mathrm{CO}_{2}$ as previously described (9). The effect of drug treatment was studied in paired kidneys obtained from the same fetus (i.e., the left kidney was incubated with media and the right kidney with Ang II). Time-lapse images of $\mathrm{GFP}^{+}$ metanephroi from Foxd ${ }^{+/+} ; \mathrm{Hoxb}^{\mathrm{GHP}+}(n=4$ fetuses) were obtained at dissection on E12.5 and after $24 \mathrm{~h}$ of culture, and those of Foxd $1^{-1}$ ${ }^{-} ;$Hoxb $7^{\mathrm{GFP}+}$ embryos were obtained at dissection on E11.5 $(n=3$ fetuses) or E12.5 ( $n=4$ fetuses) and after $24 \mathrm{~h}$ of culture; the number of UB tips was counted at each time point. Images were acquired directly from the plates using an Olympus IX70 inverted phasecontrast microscope (Pittsburgh, PA, USA), Olympus MagnaFire FW camera (Pittsburgh, PA, USA) and processed with Adobe PhotoShop 7.0.

\section{Quantitative Real-Time Reverse-Transcription Polymerase Chain Reaction}

Quantitative real-time SYBR Green RT-PCR (SABiosciences, Frederick, MD) was utilized to determine the expression of renin, ACE, and Ang $\mathrm{AT}_{1} \mathrm{R}$ mRNA levels in Foxd $1^{-1-}$ compared with Foxd $1^{+/+}$E14.5 metanephroi. The $\mathrm{AT}_{1} \mathrm{R}$ primers used detect the $\mathrm{AT}_{1 \mathrm{~A}} \mathrm{R}$ subtype. The quantity of target mRNA expression was normalized by that of GAPDH mRNA expression. E14.5 metanephroi were pooled from Foxd1 $1^{-1-}$ and Foxd1 $1^{+/+}$fetuses, and two pooled RNA samples per Foxd1 genotype were analyzed in triplicate in each run. PCR reaction was performed three times.

\section{Western Blot Analysis}

Mouse embryonic kidneys were pooled and homogenized in cold lysis buffer containing a cocktail of enzyme inhibitors. The samples were centrifuged, and the supernatants containing proteins $(30 \mu \mathrm{g} /$ lane) were resolved on 10\% SDS-polyacrylamide gels and transferred to nitrocellulose membranes. After blocking nonspecific binding, the membranes were incubated with a rabbit anti-AGT polyclonal antibody (1:100, generously provided by J. Chan, University of
Montreal), an antirenin rabbit polyclonal antibody (Santa Cruz, Dallas, TX, USA sc-22752, 1/200), a rabbit polyclonal anti-Foxd1 antibody (Santa Cruz, sc-133586, 1:200), and an anti- $\beta$-actin monoclonal antibody (Sigma, St Louis, MO; 1:5000) at room temperature for $1 \mathrm{~h}$. After washes in phosphate-buffered saline/ Tween, the nitrocellulose membrane was exposed for $1 \mathrm{~h}$ at room temperature to the secondary antibody. Immunoreactive bands were visualized using the enhanced chemiluminescence detection system (ECL, Amersham, NJ).

\section{Immunohistochemistry for AGT and Renin}

Immunolocalization of AGT and renin proteins was performed in Foxd $1^{-1-}$ and Foxd1 $1^{+/+}$E17.5 metanephroi ( $n=3$ per group). Immunostaining was performed with the immunoperoxidase technique using the Vectastain Elite kit (Vector Laboratories, Burlingame, CA). A renin rabbit polyclonal antibody was generously provided by Ariel Gomez, University of Virginia, and used at a dilution of 1:500 (ref. 10). Specificity of immunostaining was documented by the elimination of immunoreactivity after omission of the primary antibody. Spatial colocalization of AGT immunoreactivity in the proximal tubules was achieved by co-staining with fluorescein isothiocyanate-conjugated lotus tetragonolobus lectin agglutinin (LTA; Vector Laboratories, 1:100). The number of LTApositive tubular structures was counted in a blinded manner per kidney section ( $n=3$ mice per genotype, two sections per kidney). The number of AGT-positive structures in LTA-positive tubules was counted by colocalization of red staining (AGT) with green staining (LTA) in the same kidney sections.

\section{Foxd1 Overexpression in MK4 Cells}

MK4 cells (generously provided by S. Potter, University of Cincinnati) were plated into six-well plates at $2 \times 10^{5}$ cells per well and grown overnight in Dulbecco's modified essential medium highglucose media with $10 \% \mathrm{FBS}$ at $37^{\circ} \mathrm{C}$ and $5 \% \mathrm{CO}_{2}$ to $\sim 70 \%$ confluence. The cells were then incubated with $1 \mu \mathrm{g}$ of Foxd1 expression vector (CMV-BF2; $n=3$ wells; a kind gift from Leif Oxburgh, Maine Medical Center Research Institute) (11), with a MOCK plasmid pcDNA ${ }^{\mathrm{TM}_{3}} 3$ (Thermo Fisher Scientific, Grand Island, NY, USA), or without Foxd1 expression vector ( $n=3$ wells) but using Lipofectamine LTX with PLUS mixture (Invitrogen, Grand Island, NY, USA) at $37^{\circ} \mathrm{C}$ for $24 \mathrm{~h}$. Non-transfected cells were selectively eliminated by the addition of gentamicin $(100 \mu \mathrm{g} / \mu \mathrm{l}$ per 


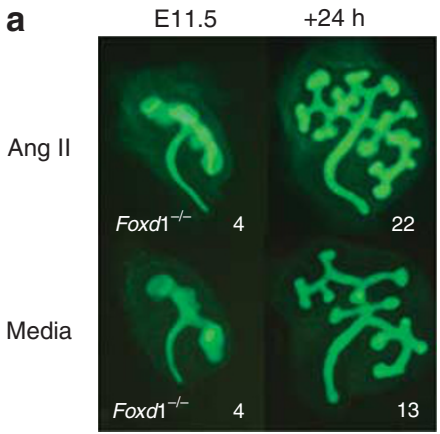

C

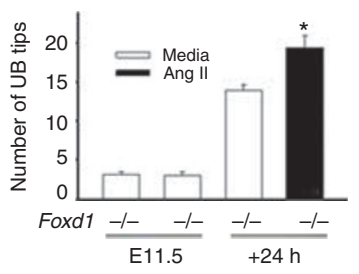

d

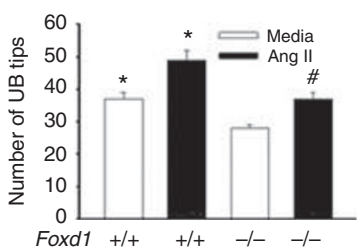

b
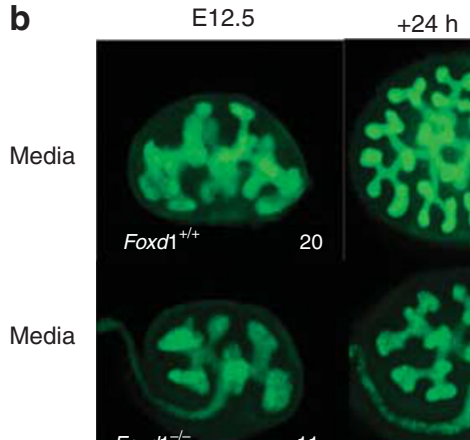

Ang II

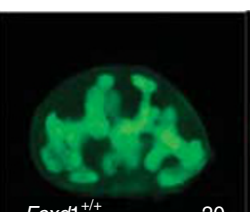

20 $+24 \mathrm{~h}$

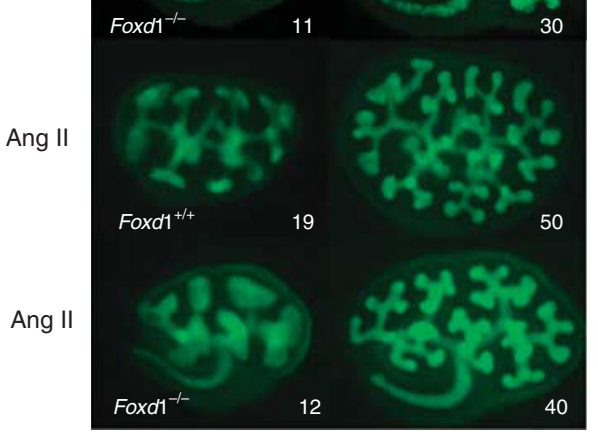

Figure 1. Effect of genetic inactivation of Foxd1 and treatment with angiotensin (Ang) II on ureteric bud (UB) branching in mouse metanephroi. Time-lapse images of Foxd $1^{-/-} / \mathrm{Hoxb} 7^{\mathrm{GFP}+}$ kidneys isolated on E11.5 and grown ex vivo for $24 \mathrm{~h}$ in the presence of Ang II (10 ${ }^{-5} \mathrm{M}$ ) or media (control; a). (b) Time-lapse images of Foxd $1^{-/-} / \mathrm{Hoxb} 7^{\mathrm{GFP}+}$ and Foxd $1^{+/+} / \mathrm{Hoxb} 7^{\mathrm{GFP}+}$ metanephroi isolated on E12.5 and grown ex vivo in the presence of Ang II or media (control; b). UBs are visualized with GFP (green; a,b). Bar graph showing the number of UB tips in Foxd ${ }^{-/-} /$Hoxb7 $^{\mathrm{GFP}+}$ metanephroi on E11.5 and after $24 \mathrm{~h}$ of culture. ${ }^{*} P<0.05$ vs. media (c). Bar graph showing the effect of Ang II or media (control) on UB tip number in E12.5 Foxd $1^{-1-} / \mathrm{Hoxb} 7^{\mathrm{GFP}+}$ and Foxd $1^{+/+} / \mathrm{Hoxb} 7^{\mathrm{GFP}+}$ metanephroi after $24 \mathrm{~h}$ of ex vivo culture (d; ${ }^{* P}<0.01$ vs. Foxd $1^{-/-}+$media; ${ }^{\#} P<0.05$ vs. Foxd $1^{-/}$ ${ }^{-}+$media). Numbers on $(\mathbf{a}, \mathbf{b})$ indicate the number of UB tips. GFP, green fluorescent protein.

well). Transfected cells were harvested for RNA or protein extraction at $\sim 90 \%$ confluence. The experiments were performed twice. Protein concentration was measured using a Pierce BCA Protein Concentration Kit (Thermo Scientific). The effect of Foxd1 transfection on Foxd1 protein expression was determined by western blot analysis and on renin, AGT, ACE, and $\mathrm{AT}_{1} \mathrm{R}$ mRNA levels by real-time SYBR Green RT-PCR. All samples were blind-coded for counting. All experiments involving mice were approved by Tulane Institutional Animal Care and Use Committee.

\section{Statistical Analysis}

Data are presented as mean \pm SEM. Differences among the treatment groups in mRNA levels and the number of UB tips were analyzed by one-way ANOVA followed by Bonferroni's test. Differences among the treatment groups in protein levels were analyzed by Student's $t$-test. A $P$ value $<0.05$ was considered statistically significant.

\section{RESULTS}

\section{Effect of Foxd1 Gene Dosage and Ang II on UB Branching}

The number of UB tips was reduced in Foxd $1^{-/-} ;$Hox $b 7^{\mathrm{GFP}+}$ ( $n=4$ fetuses, eight kidneys) compared with Foxd1 ${ }^{+/+}$; Hoxb $7^{\mathrm{GFP}+}$ ( $n=4$ fetuses, eight kidneys) E12.5 metanephroi $(14 \pm 2.1$ vs. $22 \pm 1.2, P<0.05$; Figure 1). These findings indicate that Foxd1 signaling stimulates early UB branching and are consistent with previous reports that implicate Foxd1 in renal branching morphogenesis (7). To further investigate the functional cross-talk between RAS and Foxd1, we examined the ability of exogenous Ang II to rescue UB morphogenesis in Foxd $1^{-1-}$ mice. We previously demonstrated that treatment of whole intact E12.5 mouse metanephroi grown ex vivo with $10^{5} \mathrm{M}$ Ang II increases the number of UB tips compared with treatment with control media (12). We therefore used the same concentration of Ang II in this study. Following $24 \mathrm{~h}$ of ex vivo culture, the UB tip number remained reduced in Foxd $1^{-1-}$ compared with that in Foxd $1^{+/+}$kidneys grown in the presence of media (control; Figure 1). Treatment with Ang II increased the UB tip number in both Foxd $1^{-/-}(37 \pm 1.8$ vs. $28 \pm 11, P<0.05)$ and Foxd $1^{+/+}(48 \pm 2$ vs. $38 \pm 1.8, P<0.01)$ kidneys compared with control (Figure 1). Ang II caused a similar percentage increase in the UB tip number in Foxd $1^{-1-}(n=4$ kidneys $)$ and Foxd $1^{+/+}$( $n=4$ kidneys $)$ metanephroi after $24 \mathrm{~h}$ of culture $(135 \pm 9 \%$ vs. $139 \pm 7 \%, P=0.87)$. To determine whether there is a true restoration of UB branching in Ang II-treated Foxd $1^{-/-}$metanephroi, we investigated the effect of Ang II on UB tip number in kidneys isolated from E11.5 Foxd $^{-/-}$mice and grown ex vivo for $24 \mathrm{~h}$ with and without Ang II. Although the initial number of UB tips did not differ on E11.5 (3.2 \pm 0.3 vs. $3.1 \pm 0.6, P=0.8)$, treatment with Ang 


\section{Articles | song et al.}

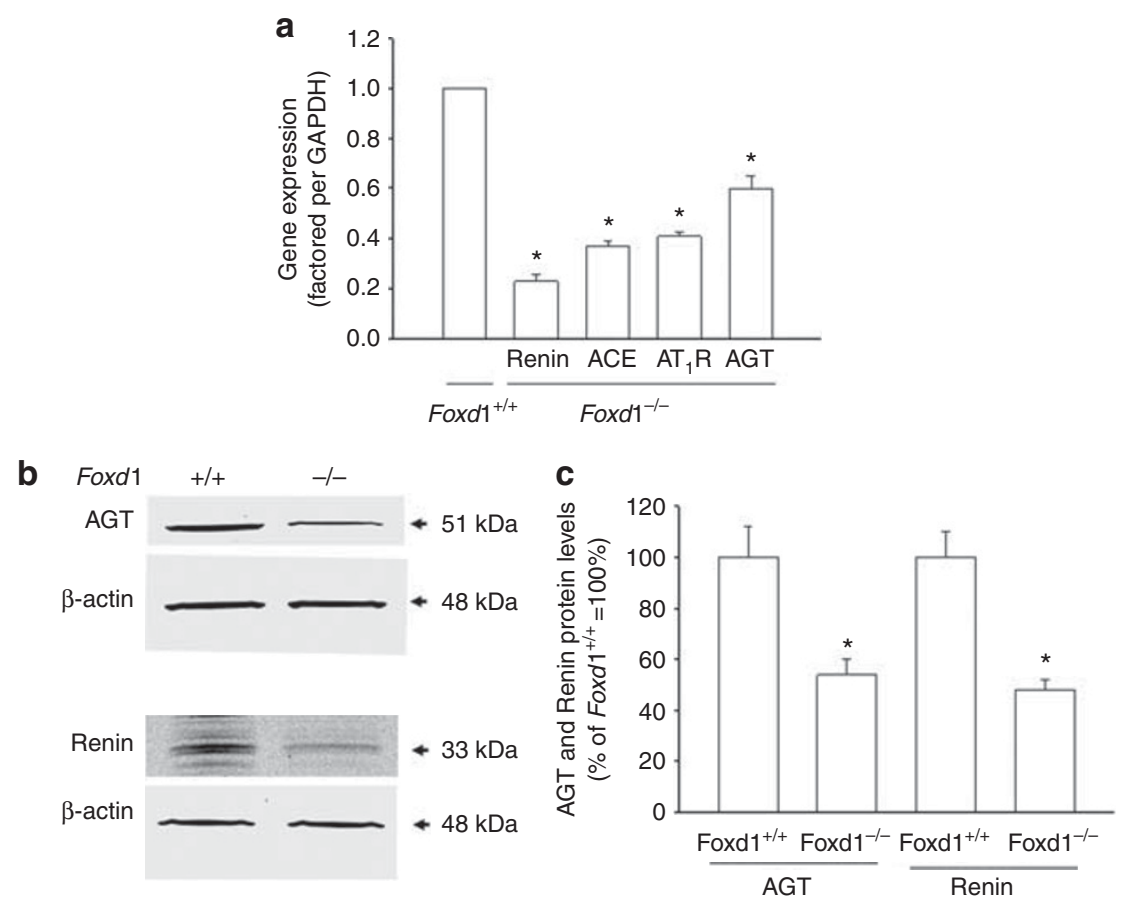

Figure 2. Effect of genetic inactivation of Foxd1 on RAS gene and protein expression in the E14.5 metanephroi. Bar graph showing the effect of genetic inactivation of Foxd1 on AGT, renin, ACE, and Ang II AT ${ }_{1} \mathrm{R}$ mRNA expression factored per expression of GAPDH mRNA in the mouse kidney as determined by qRT-PCR (a; Foxd $1^{+/+}$kidney values are normalized to 1 , and data are presented as relative-fold difference. ${ }^{*} P<0.05$ vs. Foxd $1^{+/+}$). Western blots (30 $\mu \mathrm{g}$ total protein/lane) showing angiotensinogen (AGT) and renin protein expression in Foxd $1^{+/+}$and Foxd $1^{-/-}$kidneys (b). Bar graph showing that $A G T / \beta$-actin and renin/ $\beta$-actin ratios are decreased in Foxd $1^{-/-}$compared with Foxd $1^{+/+}$metanephroi $\left(\mathbf{c} ;{ }^{*} P<0.05\right.$ vs. Foxd $1^{+/+}$). ACE, angiotensin I-converting enzyme; Ang, angiotensin.

II increased the number of UB tips $(19 \pm 1.4$ vs. $14 \pm 0.6$, $P<0.05)$ compared with the number after treatment with control media (Figure 1). This effect of Ang II is consistent with the ability of Foxd1 to function upstream of RAS.

\section{Effect of Foxd1 Gene Dosage on AGT, Renin, ACE, and $A T_{1} R$ Gene Expression and on AGT and Renin Protein Expression in the Embryonic Kidney}

To determine whether lack of Foxd1 in Foxd1 ${ }^{-1-}$ mice that manifest impaired UB branching downregulates AGT, renin, $\mathrm{ACE}$, and Ang II $\mathrm{AT}_{1} \mathrm{R}$ gene expression in the developing kidney in vivo, we measured $\mathrm{AGT}$, renin, $\mathrm{ACE}$, and $\mathrm{AT}_{1} \mathrm{R}$ mRNA levels in Foxd1 ${ }^{-1-}$ and Foxd1 ${ }^{+/+}$E14.5 mouse metanephroi. Renin $(0.23 \pm 0.01$ vs. $1.0 \pm 0, P<0.01)$, AGT $(0.61 \pm 0.02$ vs. $1.0 \pm 0, P<0.01), \operatorname{ACE}(0.37 \pm 0.02$ vs. $1.0 \pm 0$, $P<0.01)$, and $\mathrm{AT}_{1} \mathrm{R}(0.41 \pm 0.06$ vs. $1.0 \pm 0, P<0.01) \mathrm{mRNA}$ levels were decreased in Foxd1 $1^{-1-}$ metanephroi compared with the levels in Foxd1 ${ }^{+/+}$metanephroi (Figure 2a). To determine whether the observed decrease in AGT and renin gene expression in Foxd1 ${ }^{-1-}$ kidneys is accompanied by a reduction in AGT and renin protein levels, we measured AGT and renin protein contents in E14.5 Foxd1 $1^{-1-}(n=3$ pooled samples) and Foxd1 ${ }^{+/+}$( $n=3$ pooled samples) metanephroi by western blot analysis. Both AGT ( $54 \pm 6 \%$ vs. $100 \pm 12 \%$, $P<0.01)$ and renin $(48 \pm 4 \%$ vs. $100 \pm 11 \%, P<0.01)$ protein levels were decreased in Foxd1 ${ }^{-1-}$ compared with Foxd1 $1^{+/+}$ metanephroi (Figure $\mathbf{2 b}, \mathbf{c}$ ).
To delineate the effect of Foxd1 gene dosage on the spatial expression of AGT and renin proteins in the embryonic kidney, we mapped the cellular localization of AGT and renin proteins in E17.5 Foxd1 ${ }^{-1-}(n=3)$ and Foxd1 ${ }^{+/+}(n=3)$ metanephroi by immunohistochemistry. Consistent with a smaller surface area of the UB tree observed on E12.5 (Figure 1b), the kidney size was grossly reduced in Foxd1 $1^{-/-}$ compared with that in Foxd1 $1^{+++}$mice on E17.5 (Figure 3a,b). In Foxd1 ${ }^{+/+}$kidneys, AGT immunoreactivity was observed in tubules that resemble morphologically proximal tubules and in UB branches (Figure 3b). Overall, less AGT immunoreactivity was observed in Foxd1 ${ }^{-1-}$ than in Fox $^{+/+} 1^{++}$metanephroi, although AGT staining appeared to be present in the same structures as in the wild-type kidneys (Figure 3a,b). The number of AGT-positive structures in the proximal tubules visualized with anti-LTA antibody was lower $(10 \pm 2.1$ vs. $29 \pm 4.1, P<0.05)$, whereas the number of LTApositive proximal tubules was not different $(90 \pm 8.1$ vs. $99 \pm 9.5, P=0.50)$, in Foxd1 $1^{-1-}$ compared with Foxd1 $1^{+/+}$ metanephroi (Figure 3d,e). These findings suggest that reduced AGT expression in the proximal tubules in Foxd1 $1^{-1-}$ metanephroi is not secondary to a decrease in the number of proximal tubules. These findings are consistent with a previous report showing abundant proximal tubules in mice with global Foxd1 knockout (8). In addition, the number of nephrons on E18.5 in Foxd1 mutants (on a per-kidneyvolume basis) is similar to the number of nephrons present in 

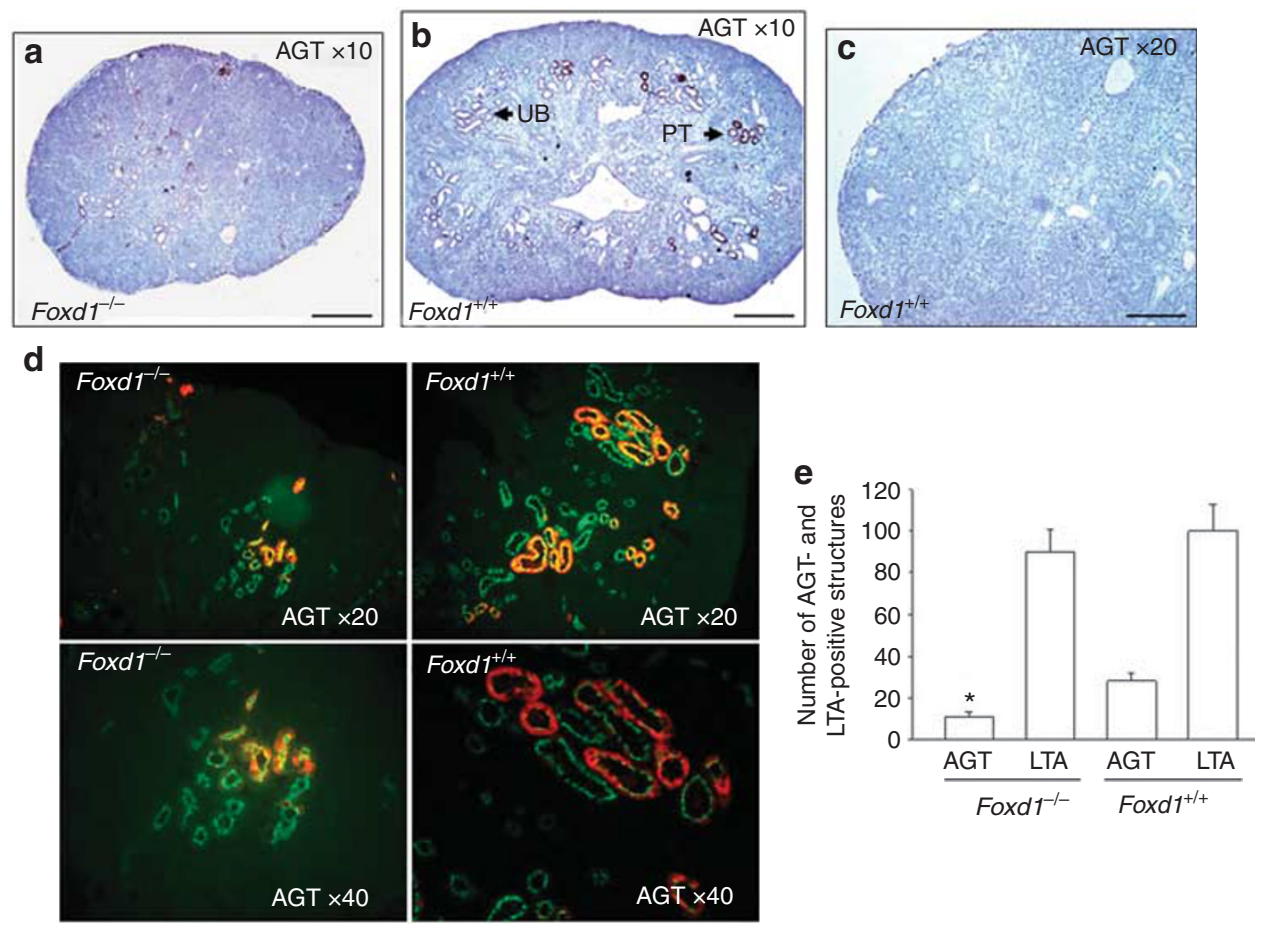

Figure 3. Immunolocalization of angiotensinogen (AGT) protein in the embryonic Foxd1 $1^{+/+}$and Foxd $1^{-/-}$kidneys on E17.5. AGT immunoreactivity (brown staining) is present in the tubules that resemble morphologically proximal tubules (PT) and in UB branches (a,b). Control section, where the addition of the primary antibody was omitted, demonstrates no brown staining (c). Spatial co-localization of FITC-conjugated AGT (red) and lotus tetragonolobus lectin agglutinin (LTA, green) immunoreactivity in the proximal tubules in Foxd $1^{+/+}$and Foxd $1^{-/-}$metanephroi (d). Bar graph showing the number of AGT- and LTA-positive structures in Foxd $1^{-/-}$and Foxd $1^{+/+}$metanephroi $\left(\mathbf{e} ;{ }^{*} P<0.05\right.$ vs. Foxd $\left.{ }^{+/+}\right)$. FITC, fluorescein isothiocyanate. Scale bar $=100 \mu \mathrm{m}$.
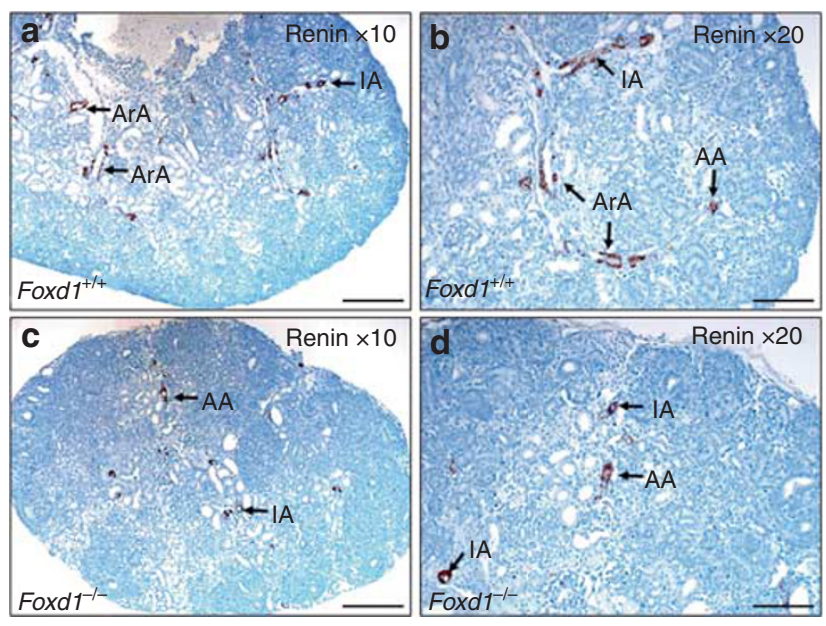

Figure 4. Immunolocalization of renin protein in the fetal Foxd $1^{+/+}$and Foxd $1^{-1-}$ kidneys on E17.5. Renin immunoreactivity (brown staining) is present in the afferent arterioles (AA) and interlobular (IA) and arcuate $(\operatorname{Ar} A)$ arteries $(\mathbf{a}, \mathbf{b})$. In Foxd $1^{-1-}$ kidneys, renin immunoreactivity is confined to a few cells in the vascular structures that resemble afferent arterioles and interlobular and arcuate arteries (c,d; scale bar $=100 \mu \mathrm{m})$.

the wild-type kidney (8). Lack of reduction in the number of LTA-positive proximal tubules in spite of reduced UB branching in Foxdl mutants may be explained by the increased ratio of pre-tubular aggregated per UB ampulla (8).
In E17.5 Foxd1 ${ }^{+/+}$kidneys, renin immunoreactivity is present throughout the length of the afferent arterioles and interlobular and arcuate arteries (Figure 4a,b). In Foxd1 $1^{-1-}$ kidneys, renin immunoreactivity is less broad and is confined to a few cells in vascular structures that resemble morphologically afferent arterioles and interlobular and arcuate arteries (Figure $\mathbf{4 c , d}$ ). Whether the decrease in renin immunostaining is due to a direct effect on renin (or another gene) or due to a general decrease in kidney growth remains to be determined. Collectively, these data suggest that endogenous Foxd1 acts upstream of RAS to increase $\mathrm{AGT}$, renin, $\mathrm{ACE}$, and $\mathrm{AT}_{1} \mathrm{R}$ mRNA expression, as well as AGT and renin protein levels.

\section{Effect of Foxd1 Overexpression in Mesenchymal Cells on RAS Gene Expression}

To determine whether Foxd1 overexpression in renal mesenchymal cells upregulates $A G T$, renin, $A C E$, and $A_{1} R$ gene expression, we examined $A G T$, renin, $A C E$, and $\mathrm{AT}_{1} \mathrm{R}$ mRNA levels in MK4 cells transfected ( $n=3$ wells) or not ( $n=3$ wells) with Foxd1 expression plasmid in vitro. The MK4 cell line represents embryonic metanephric mesenchyme undergoing epithelial conversion. Notably, cultured MK4 cells maintain the expression of endogenous AGT, renin, $\mathrm{ACE}, \mathrm{AT}_{1} \mathrm{R}$, and Foxd1 mRNA (Figure 5a). Transfection of MK4 cells with Foxd1 expression plasmid increased Foxd1 


\section{Articles | song et al.}
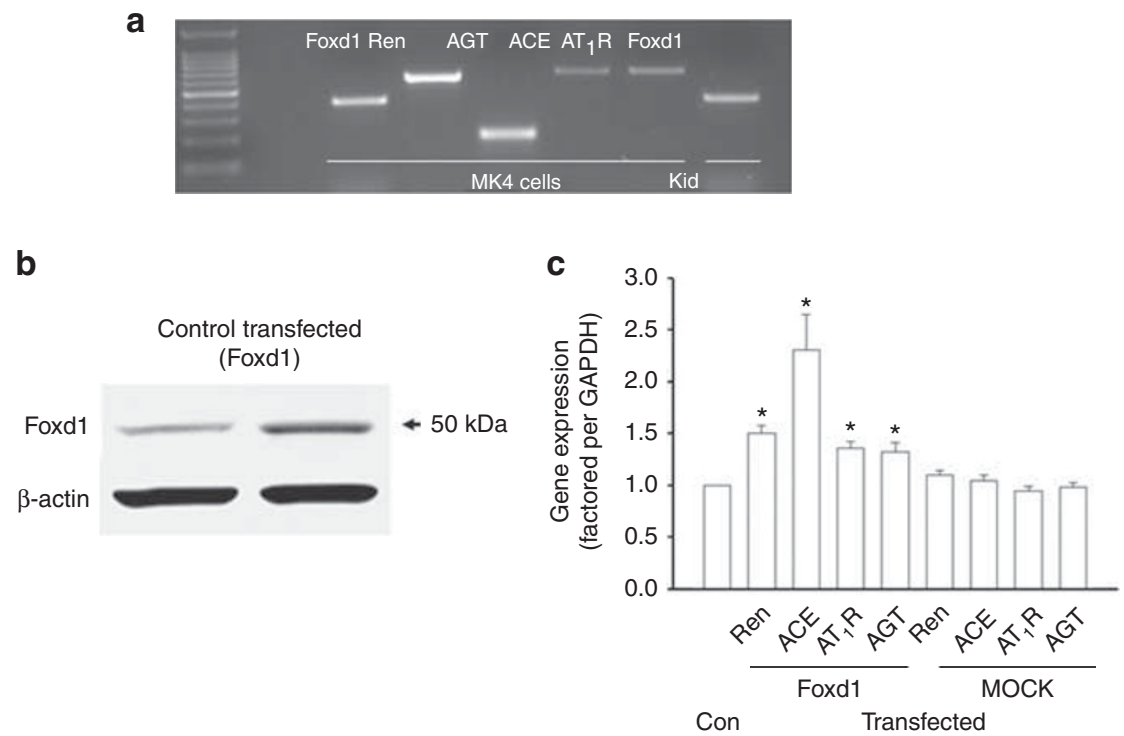

Figure 5. Mesenchymal (MK4) cells cultured in vitro express angiotensinogen (AGT, 280 bp), renin (Ren, 600 bp), angiotensin-converting enzyme (ACE, 614 bp), AT 1 R (603 bp), and Foxd1 (398 bp) mRNA (a; Kid-E14.5 mouse kidney). Western blot (30 $\mu \mathrm{g}$ total protein/lane) showing increased Foxd1 protein levels in MK4 cells transfected with Foxd1 expression vector compared with control untransfected cells (b). Bar graph shows the effect of Foxd1 overexpression in MK4 cells (Foxd1) on renin (Ren), AGT, ACE, and AT 1 R mRNA expression as determined by qRT-PCR (c; Con, control MK4 cells not transfected with Foxd1 vector. MOCK, MK cells transfected with a MOCK plasmid that does not express Foxd1). ${ }^{*} P<0.05$ vs. control. ACE, angiotensin I-converting enzyme.

protein levels compared with the levels in untransfected cells (Figure $5 \mathbf{b}$ ). This was accompanied by increased AGT $(1.3 \pm 0.08$ vs. $1.0 \pm 0, P<0.05)$, renin $(1.3 \pm 0.08$ vs. $1.0 \pm 0$, $P<0.05)$, ACE $(2.2 \pm 0.3$ vs. $1.0 \pm 0, P<0.01)$, and $\mathrm{AT}_{1} \mathrm{R}$ $(1.3 \pm 0.04$ vs. $1.0 \pm 0, P<0.01)$ mRNA levels in transfected compared with untransfected cells (Figure 5c). In contrast, transfection of MK4 cells with a MOCK plasmid ( $n=3$ wells) did not alter $\mathrm{AGT}$, renin, $\mathrm{ACE}$, or $\mathrm{AT}_{1} \mathrm{R}$ mRNA levels compared with the levels in untransfected cells (Figure 5c). These findings indicate that upregulation of AGT, renin, $\mathrm{ACE}$, or $\mathrm{AT}_{1} \mathrm{R}$ mRNA levels by Foxd1 expression plasmid is not due to unspecific effects. Thus, Foxd1 acts upstream of $A G T$, renin, $A C E$, and $A T_{1} R$ genes to upregulate their expression in embryonic mesenchymal cells.

\section{DISCUSSION}

The present study tested the hypothesis that Foxd1 is an upstream regulator of RAS during UB-branching morphogenesis. Our major findings are as follows: (i) the reduced UB branching observed in Foxd1 ${ }^{-1-}$ metanephroi is accompanied by decreased expression of AGT, renin, $\mathrm{ACE}$, and $\mathrm{AT}_{1} \mathrm{R}$ mRNA as well as AGT and renin protein levels; (ii) Foxd1 overexpression in mesenchymal cells increases AGT, renin, $\mathrm{ACE}$, and $\mathrm{AT}_{1} \mathrm{R}$ mRNA levels; (iii) treatment with exogenous Ang II causes a similar induction of UB branching in Foxd1 ${ }^{-1-}$ and $\mathrm{Foxd1}^{+/+}$whole intact metanephroi grown ex vivo.

Foxd1 and RAS are both critically important for metanephric kidney organogenesis $(7,8,13)$. In the mouse, Foxd1, a forkhead box transcription factor, is expressed from the onset of UB branching around Pax2-expressing cells of the induced nephrogenic mesenchyme. From E14.5 onward, Foxd1 is localized in the cortical renal stroma, with lower levels present in the medullary stroma (7). Foxd1-mutant mice exhibit impaired UB branching, aberrant nephrogenesis, and fused pelvic kidneys, a phenotype reminiscent of the horseshoe kidney, the most common renal fusion anomaly observed in humans $(7,8,14)$. Thus, lack of Foxd1 causes a defect in the ability of the stroma to support normal UB branching and nephrogenesis.

Foxd1 and RAS are essential for UB-branching morphogenesis. Reduced UB branching is observed in $A G T^{-1-}$ and Ang II $A T_{2} R^{-1-}$ mice or during pharmacologic inhibition of Ang II $\mathrm{AT}_{1} \mathrm{R}$ in embryonic mouse metanephroi grown ex vivo $(15,16)$. Using immunohistochemical analysis, we previously demonstrated that $A G T$ and $A_{1} R$ proteins are expressed in both the UB and the surrounding stroma as early as E12 in mice (5). Given that renin-producing cells originate from the mesenchyme on E11-E12, at a time when UB branching is just beginning (6), Ang II may be generated locally in the mesenchyme to act in a paracrine manner on the adjacent $\mathrm{AT}_{1} \mathrm{R}$-expressing UBs to stimulate branching. In accordance with this hypothesis, treatment of E11.5 mouse intact isolated UBs grown in three-dimentional collagen matrix gels with Ang II stimulates UB branching (17). Similarly, $\mathrm{AT}_{1} \mathrm{R}$ present in the stroma may be important in mediating stromal Ang II signaling to stimulate UB branching (5). One possible pathway may involve Ang II-induced stimulation of stromal factor fibroblast growth factor 7 (FGF7) and its coupling with the FGF7 receptor expressed on the UB (18). This possibility 


\section{Foxd1 regulates renin system Articles}

is supported by the ability of Ang II to increase bFGF mRNA levels in luteal cells (19).

Potential interactions among AGT, renin, and Foxd 1 in the stroma are supported by the findings that $A G T$ and renin promoters contain a putative binding site for Foxd1 (ref. 20). It has been shown that Foxd1 binds to a hepatocyte nuclear factor 3 (HNF3, a winged helix transcription factor) site to activate transcription (20). As mouse renin (GTAAACA, position - 736) and AGT (TGCTTGTCTAG, position - 758) genes contain a conserved HNF3 site/core sequence and HNF3B stimulates $A G T$ gene transcription (21), it may be possible that, as a transcription factor, Foxd1 stimulates the production of $\mathrm{AGT}$, renin, or $\mathrm{AT}_{1} \mathrm{R}$ in the stroma to enhance UB branching. Thus, Foxd1 may act upstream from AGT or renin, and UB-branching defects in Foxd1-mutant mice may be secondary, in part, to dysregulation of the RAS. Reduction in proximal tubule AGT protein levels in Foxd1 ${ }^{-/-}$kidneys may also be due to disruption of Foxd1-dependent signals that mediate the communication between the stromal and nephrogenic compartments. One potential signal may be stromally expressed $\beta$-catenin, reported to regulate the induction of nephrogenic progenitors (22). In support of this possibility, bioinformatic analyses reveal the presence of the consensus T-cell factor-binding sites in the regulatory regions of the AGT gene (23).

Given that RAS gene expression was not completely attenuated in the absence of Foxd1, it is possible that Foxd1 forms a complex with other transcription factors and acts indirectly to modulate the RAS gene expression. This possibility is supported by the findings that forkhead transcription factors associate with the Groucho co-repressor (24). In addition, Foxd1 associates with a Smad-binding partner, FAST-2 (25). Notably, initiation of nephron progenitor cell differentiation, normally induced by Smad signaling, is inhibited in Foxd1 ${ }^{-1-}$ kidneys(11).

One mechanism by which RAS can mediate cross-talk between stromal Foxd1 and UB is via Ret. This possibility is supported by the ability of Ang II to induce tyrosine phosphorylation of Ret in whole intact E12.5 mouse metanephroi grown ex vivo (17). Our present findings that Ang II stimulates UB branching equally in Foxd1 $1^{-1-}$ and Foxd1 $^{+/+}$mice indicate that Ang II signaling to the UB in Foxd1 mutants is intact and is consistent with the ability of Foxd1 to function upstream of RAS. Another possibility is that Foxd1-positive stromal cells give rise to renin-producing juxtaglomerular cells (26). This possibility is supported by our present findings of limited expression of renin in maturing renal vasculature in Foxd1 $1^{-1-}$ mice.

In summary, the present study demonstrates that genetic inactivation of Foxd1 in mice downregulates $A G T$, renin, $A C E$, and $A T_{1} R$ gene expression in the developing metanephroi in vivo. In contrast, Foxd1 overexpression in renal mesenchymal cells in vitro upregulates the expression of $A G T$, renin, $A C E$, and $A T_{1} R$ genes. Ang II stimulates UB branching equally in Foxd1 $1^{-1-}$ and Foxd1 $1^{+/+}$ metanephroi grown ex vivo. We conclude that Foxd1 is an upstream positive regulator of RAS during early metanephric development and propose that the cross-talk between RAS and Foxd1 is essential in UB-branching morphogenesis.

\section{ACKNOWLEDGMENTS}

This work was supported by NIH Grant DK-71699 (I.Y.). We thank Frank Costantini (Columbia University Medical Center) for providing Hoxb $7^{\mathrm{GFP}+}$, Leif Oxburgh (Maine Medical Center Research Institute) for the Foxd1 expression plasmid, and Shao-Ling Zhang (University of Montreal) for the angiotensinogen antibody.

Disclosure: The authors declare no conflict of interest.

\section{REFERENCES}

1. Costantini F, Kopan R. Patterning a complex organ: branching morphogenesis and nephron segmentation in kidney development. Dev Cell 2010;18:698-712.

2. Kriz W. Structural organization of the renal medulla: comparative and functional aspects. Am J Physiol 1981;241:R3-16.

3. Lemley KV, Kriz W. Anatomy of the renal interstitium. Kidney Int 1991;39:370-81.

4. Gribouval O, Gonzales M, Neuhaus T. Mutations in genes in the reninangiotensin system are associated with autosomal recessive renal tubular dysgenesis. Nat Genet 2005;37:964-8.

5. Iosipiv IV, Schroeder M. A role for angiotensin II AT1 receptors in ureteric bud cell branching. Am J Physiol Renal Physiol 2003;285:F199-207.

6. Sequeira Lopez ML, Pentz ES, Robert B, et al. Embryonic origin and lineage of juxtaglomerular cells. Am J Physiol Renal Physiol 2001;281: F345-56.

7. Hatini V, Huh SO, Herzlinger D, et al. Essential role of stromal mesenchyme in kidney morphogenesis revealed by targeted disruption of Winged Helix transcription factor BF-2. Genes Dev 1996;10:1467-78.

8. Levinson RS, Batourina E, Choi C, et al. Foxd1-dependent signals control cellularity in the renal capsule, a structure required for normal renal development. Development 2005;132:529-39.

9. YosypivIV, Schroeder M, El-Dahr SS. AT1R-EGFR crosstalk regulates ureteric bud branching morphogenesis. J Am Soc Nephrol 2006;17: $1005-4$.

10. Gomez RA, Pentz ES, Jin X, et al. CBP and p300 are essential for renin cell identity and morphological integrity of the kidney. Am J Physiol Heart Circ Physiol 2009;296:H1255-62.

11. Fetting JL, Guay JA, Karolak MJ, et al. FOXD1 promotes nephron progenitor differentiation by repressing decorin in the embryonic kidney. Development 2014;141:17-27.

12. Song R, Spera M, Garrett C, et al. Angiotensin II-induced activation of c-Ret signaling is critical in ureteric bud branching morphogenesis. Mech Dev 2010;127:21-7.

13. Yosypiv IV. Renin-angiotensin system in ureteric bud branching morphogenesis: insights into the mechanisms. Pediatr Nephrol 2011;26:1499-512.

14. Song R, Yosypiv IV. Genetics of congenital anomalies of the kidney and urinary tract. Pediatr Nephrol 2011;26:353-64.

15. Song R, Spera M, Garrett C, et al. Angiotensin II AT2 receptor regulates ureteric bud morphogenesis. Am J Physiol Renal Physiol 2010;298: F807-17.

16. Song R, Van Buren T, Yosypiv IV. Histone deacetylases are critical regulators of the renin-angiotensin system during ureteric bud branching morphogenesis. Pediatr Res 2010;67:573-8.

17. Song R, Preston G, YosypivIV. Angiotensin II stimulates in vitro branching morphogenesis of the isolated ureteric bud. Mech Dev 2011;128:359-67.

18. Qiao J, Uzzo R, Obara-Ishihara T, et al. FGF-7 modulates ureteric bud growth and nephron number in the developing kidney. Development 1999;126:547-54.

19. Stirling D, Magness RR, Stone R, et al. Angiotensin II inhibits luteinizing hormone-stimulated cholesterol side chain cleavage expression and 


\section{Articles | song et al.}

stimulates basic fibroblast growth factor expression in bovine luteal cells in primary culture. J Biol Chem 1990;265:5-8.

20. Zhang H, Palmer R, Gao X, et al. Transcriptional activation of placental growth factor by the forkhead/winged helix transcription factor Foxd1. Cur Biol 2003;13:1625-9.

21. Cui Y, Narayanan CS, Zhou J, et al. Exon-I is involved in positive as well as negative regulation of human angiotensinogen gene expression. Gene 1998;224:97-107.

22. Boivin FJ, Sarin S, Lim J, et al. Stromally expressed $\beta$-catenin modulates Wnt9b signaling in the ureteric epithelium. PLoS ONE 2015;10:e0120347.

23. Zhou L, Li Y, Hao S, et al. Multiple genes of the renin-angiotensin system are novel targets of wnt/beta-catenin signaling. J Am Soc Nephrol 2015;26:107-20.

24. Yaklichkin S, Vekker A, Stayrook S, Lewis M, Kessler DS. Prevalence of the EH1 Groucho interaction motif in the metazoan Fox family of transcriptional regulators. BMC Genomics 2007;8:201.

25. Dou C, Lee J, Liu B, Liu F, Massague J, Xuan S, Lai E. BF-1 interferes with transforming growth factor beta signaling by associating with Smad partners. Mol Cell Biol 2000;20:6201-11.

26. Sequeira Lopez ML, Gomez RA. Development of the renal arterioles. J Am Soc Nephrol 2011;22:2156-65.

27. Miyazaki Y, Oshima K, Fogo A, et al. Bone morphogenic protein 4 regulates the budding site and elongation of the mouse ureter. J Clin Invest 2000;105:863-73.

28. Mendelsohn C, Batourina E, Fung S, et al. Stromal cells mediate retinoiddependent functions essential for renal development. Development 1999;126:1139-48.
29. Quaggin SE, Schwartz L, Cui S, et al. The basic helix-loop-helix protein Pod1 is critically important for kidney and lung organogenesis. Development 1999;126:5771-83.

30. Schnabel CA, Selleri L, Cleary ML. Pbx1 is essential for adrenal development and urogenital differentiation. Genesis 2003;37:123-30.

31. Lin Y, Liu A, Zhang S, et al. Induction of ureter branching as a response to Wnt-2b signaling during early kidney organogenesis. Dev Dynamics 2001;222:26-39.

32. Nagata M, Tanimoto K, Fukamizu A, et al. Nephrogenesis and renovascular development in angiotensinogen-deficient mice. Lab Invest 1996;75:745-53.

33. Oliverio MI, Kim HS, Ito M, et al. Reduced growth, abnormal kidney structure, and type 2 (AT2) angiotensin receptor-mediated blood pressure regulation in mice lacking both AT1A and AT1B receptors for angiotensin II. Proc Natl Acad Sci USA 1998;95:15496-501.

34. Miyazaki Y, Tsuchida S, Fogo A, et al. The renal lesions that develop in neonatal mice during angiotensin inhibition mimic obstructive nephropathy. Kidney Int 1999;55:1683-95.

35. YosypivIV, Boh MK, Spera M, et al. Downregulation of Spry-1, an inhibitor of GDNF/Ret, as a mechanism for angiotensin II-induced ureteric bud branching. Kidney Int 2008;74:1287-93.

36. Nishimura H, Yerkes E, Hohenfellner K, et al. Role of the angiotensin type 2 receptor gene in congenital anomalies of the kidney and urinary tract, CAKUT, of mice and men. Mol Cell 1999;3:1-10.

37. Oshima K, Miyazaki Y, Brock JW, et al. Angiotensin type II receptor expression and ureteral budding. J Urol 2001;166:1848-52. 